\title{
France Lymphome Espoir : une association de patients pour des patients
}

\author{
M.-F. Bacqué \\ (C) Springer-Verlag France 2012
}

Cette association a été créée le 16 février 2006. France Lymphome Espoir est une association de patients atteints d'un lymphome qui a pour objectif d'assister et d'informer tous ceux qui sont touchés par cette maladie. Guy Bouguet, président de France Lymphome Espoir, a été lui-même atteint par un lymphome et se rend régulièrement dans les congrès de cancérologie et de psycho-oncologie.

France Lymphome Espoir s'est inspirée de l'association américaine Lymphoma Research Foundation, parce qu'elle va au-delà du soutien aux patients. Elle participe à la recherche sur la maladie, elle organise des conférences sur Internet, met sur pied des rencontres avec des chercheurs et des médecins...

Avec plus de 12000 nouveaux cas de lymphome par année, ce cancer a la sixième place en termes d'incidence. En l'absence d'information en la matière, la première action de France Lymphome Espoir a été de créer un document qui explique les bases de la maladie. Édité à 5000 exemplaires, il a été envoyé dans les hôpitaux où il y a des Espaces Rencontre Information (ERI). Dans un deuxième temps, le site Internet a été développé. Ce site est magnifique, interactif, il permet de se documenter mais aussi de participer à des forums, des blogs, de regarder des vidéos sur la maladie et même de passer des QCM pour voir si l'on a bien retenu les informations ! Des bénévoles aident à mettre en place la structure, le numéro d'appel, animent des forums de parole qui ont lieu dans les hôpitaux (le premier s'est tenu le 20 septembre 2006 à l'hôpital Saint-Louis). La diffusion de l'information sur ce cancer du système lymphatique est la priorité de l'association, le soutien des malades et de leur famille, le second objectif.

France Lymphome Espoir encourage la recherche et la formation grâce à l'appui d'un comité scientifique composé de spécialistes de la maladie. L'association développe également des actions sociales pour une meilleure connaissance de la maladie, auprès de la population, des familles et des organismes sociaux.

" Clic-claques aux lymphomes », à voir sur le site, résulte d'une exposition de photos (on pense bien entendu au clic-clac de l'appareil photo) qui a permis aux participants de "mettre une claque " symbolique au lymphome. Pleine d'énergie, cette exposition stimule les malades et leur famille à s'informer, s'entourer, se soutenir. Ne ratez pas le site, il est très réussi !

France Lymphome Espoir, 1, avenue Claude-Vellefaux, F-75475 Paris cedex 10, France www.francelymphomeespoir.fr

M.-F. Bacqué $(\square)$ 